\title{
Avaliação do Programa de Residência Médica do Departamento de Pediatria da Faculdade de Medicina da Universidade de São Paulo
}

\author{
Assessment of the Medical Residency Programs \\ of the Paediatrics Department of the University \\ of São Paulo Medical School in light of the \\ Professional Profiles of the Graduates.
}

\author{
PALAVRAS CHAVE \\ - Residência Médica. \\ - Pediatria. \\ - Avaliação \\ - Egressos.
}

Recebido em: 20/10/2010

Aprovado em: 10/07/2011
Vera Hermina K. Koch Ulysses Doria Filho ${ }^{I I}$ Valdes Roberto Bollela

Introdução: A avaliação do perfil do egresso é útil para investigar o sincronismo entre o treinamento fornecido e a demanda profissional. Objetivos: Analisar, por meio de um questionário padronizado, o perfil profissional dos egressos do programa de Residência Médica (RM) de Pediatria FMUSP no período 1997-2008 e a compatibilidade entre esse perfil e a prática clínica na comunidade. Métodos: Pôde-se localizar o endereço eletrônico de 297/446 egressos; 150 responderam à pesquisa, após consentimento informado. Resultados: 126/150 respondentes do sexo feminino, idade média de 31,9 $\pm 2,8$ anos, $61,3 \%$ oriundas do Estado de São Paulo (SP), 64\% atuam profissionalmente em SP. Os egressos originários das regiões Norte, Nordeste e Centro-Oeste se estabeleceram profissionalmente em SP. A escolha da Pediatria foi relacionada a afeto pela criança, preferência de exercício profissional voltado à criança e característica generalista da especialidade. Os estágios considerados mais importantes para o exercício profissional foram na área de urgência/emergência, neonatologia, enfermarias de Pediatria geral e de especialidades pediátricas. A ampliação da RM em Pediatria para três anos foi aprovada por $72 \%, 66 \%$ destes com vistas a aumentar a exposição às especialidades pediátricas; $78 \%$ se consideraram profissionalmente realizados e $22 \%$ parcialmente realizados em virtude de renda mensal inferior à esperada e por não terem iniciado/concluído a pós-graduação; $54 \%$ exercem função docente, principalmente em serviços públicos. As expectativas se concentram no início/término da pós-graduação, aprimoramento/reconhecimento profissional e melhoria da renda, com foco em clínica privada. Conclusões: Os egressos sugerem formação mais sólida nas especialidades pediátricas e demonstram tendência a se estabelecer em SP, não retornando ao Estado de origem.

Introduction: Graduate profile assessment is a useful means of investigating the synchronicity between training provided and professional demand. Objectives: To review, by means of a standardized questionnaire, the professional profile of graduates from the Paediatric Medical Residency program of FMUSP in the period 1997-2008 and the compatibility of that profile with medical practice in the community. Methods: The email addresses of 297/446 graduates were located; 150 responded to the survey, following informed consent. Results: $126 / 150$ respondents were female, average age $31.9 \pm 2.8$ years, $61.3 \%$ from São Paulo state (SP), $64 \%$ worked professionally in SP. Graduates from the North, Northeast and Central-West regions established their careers in SP. The choice of paediatric medicine was related to affection for children, preference for working with children and the general nature of the speciality. The internships considered to be of most importance for professional practice were in the area of emergency medicine, neonatal care, general paediatric units and paediatric specialities. Extending the paediatric medical residency to three years was approved by $72 \% ; 66 \%$ of whom wished for greater contact with paediatric specialities; $78 \%$ considered themselves professionally fulfilled and $22 \%$ partially fulfilled in virtue of earning less than expected or due to lack of academic qualification; $54 \%$ worked in a teaching capacity, primarily in the public sector. Future expectations related mainly to starting/completing graduate studies, professional improvement/recognition and higher earnings, with a focus on private practice. Conclusions: The graduates suggest more solid training in paediatric specialities and display a trend of settling in SP, and not returning to their home state. 


\section{INTRODUÇÃO}

A Faculdade de Medicina da Universidade de São Paulo (FMUSP), a primeira escola médica criada no Estado de São Paulo, também foi a primeira a contar com um hospital-escola, o Hospital das Clínicas da FMUSP. Em 1944, teve início o primeiro programa de Residência Médica (RM) no Brasil ${ }^{3}$. A RM foi regulamentada pelo Decreto $\mathrm{n}^{\circ} \mathbf{8 0 . 2 8 1}$, de 5 de setembro de 1977, e constitui uma modalidade de ensino de pós-graduação destinada a médicos, sob a forma de curso de especialização, funcionando em instituições de saúde, sob a orientação de profissionais médicos de elevada qualificação ética e profissional, sendo considerada o "padrão ouro" da especialização médica. O mesmo decreto criou a Comissão Nacional de Residência Médica (CNRM).

A RM no Departamento de Pediatria da Faculdade de Medicina da Universidade de São Paulo oferece treinamento nos três níveis de atuação médica, com predomínio de atividades de atenção primária e secundária durante o primeiro ano de treinamento, focando o cuidado e atenção à criança sadia. No segundo ano, há predomínio de atividades de atenção secundária e terciária em ambiente hospitalar de maior complexidade. Os campos de prática utilizados englobam o Centro de Saúde Escola Samuel Barnsley Pessoa, para atendimento e treinamento em atenção primária, o Hospital Universitário e o Hospital Auxiliar de Cotoxó, para atendimento e treinamento em atenção secundária, e o Instituto da Criança-HCFMUSP, para atendimento e treinamento em atenção terciária.

Apesar da existência de inúmeros programas de residência médica ativos no Brasil, praticamente não há programas regulares de avaliação da qualidade dos mesmos. Para conhecer a qualidade de um programa de formação profissional, é fundamental conhecer como está se saindo o profissional treinado neste ou naquele programa. $\mathrm{O}$ acompanhamento regular e sistemático de egressos é uma prática incomum em diversas áreas, especialmente entre instituições formadoras de profissionais da saúde. Existem poucos dados, e praticamente não encontramos publicações com informações sobre o perfil dos egressos de programas de especialização e seu desempenho ao longo da carreira.

A avaliação do perfil do egresso médico por meio de questionários autoaplicáveis constitui metodologia de avaliação utilizada para levantamento das perspectivas de campo de trabalho, perfil de escolhas profissionais e condições de inserção e satisfação profissional. Este instrumento pode ser utilizado também como avaliação externa complementar de um centro formador, no sentido de investigar a adequação entre o treinamento fornecido e a real demanda ao exercício profissional apresentada pela comunidade ${ }^{5-8}$.
Apesar da utilidade desta metodologia, não há, até o momento, publicações sobre este tipo de avaliação de programas de Residência Médica em Pediatria no Brasil.

O presente estudo teve como objetivo analisar os resultados do programa de Residência Médica do Departamento de Pediatria da FMUSP com base em informações de egressos quanto ao perfil profissional e compatibilidade entre programa curricular e desafios da prática clínica na comunidade no período 1997-2008.

A realização deste estudo, no momento atual, tem importância adicional porque vai contribuir para avaliar a necessidade de ampliar a duração do atual programa de dois para três anos. Esta discussão começou a partir de uma proposição do Departamento de Pediatria da FMUSP, com apoio da Sociedade Brasileira de Pediatria.

\section{MÉTODOS}

Com base nos arquivos do Departamento de Pediatria, foram identificados 446 egressos no período de 1997 a 2008. Utilizando-se o banco de dados do Departamento de Pediatria, do Conselho Regional de Medicina de São Paulo e as redes sociais, foram levantados 297 endereços eletrônicos, aos quais foi enviado um convite que explicava o projeto e a forma de acesso ao questionário para autorresposta. $\mathrm{O}$ aceite eletrônico do consentimento informado era requerido para dar acesso ao questionário, com a garantia de sigilo e privacidade de todas as informações fornecidas. Obtivemos respostas de 150 $(33,6 \%)$ egressos.

O questionário apresentava aos respondentes questões relativas a quatro grandes temas: dados pessoais, formação profissional, formação acadêmica e momento/perspectivas atuais.

A análise estatística foi feita a partir da criação de um banco de dados criado para este fim. Depois, os dados foram submetidos a análise estatística pelo Núcleo .... (NuCAMPE), do Instituto da Criança do HCFMUSP.

As variáveis quantitativas foram descritas através de suas médias e desvios-padrão. As variáveis categóricas foram descritas por meio de frequências e porcentagens. As perguntas abertas (textos) foram analisadas através de análise de discurso e agrupadas segundo temáticas comuns.

O estudo foi aprovado em 21 de maio de 2009 pela Comissão de Ética do HCFMUSP sob o número 041/09.

\section{RESULTADOS}

Os questionários nem sempre puderam ser integralmente aproveitados, já que algumas pessoas não responderam todas as questões existentes. Por isso, existe uma variação nos números de respostas a cada pergunta. 
Dentre os 150 respondentes, 117 informaram o sexo, sendo $97(82,9 \%)$ do sexo feminino, com média de idade de 31,9 $\pm 2,82$ anos. Entre os homens, a média de idade foi de 31,7 $\pm 2,62$ anos.

O Estado de São Paulo foi declarado como Estado de origem de 90/119 (75,6\%) egressos, sendo os demais $29(24,4 \%)$ originários de outros 12 Estados (Gráfico 1).

\section{GRÁFICO 1}

Origem dos egressos de Pediatria que responderam a esta questão (119)

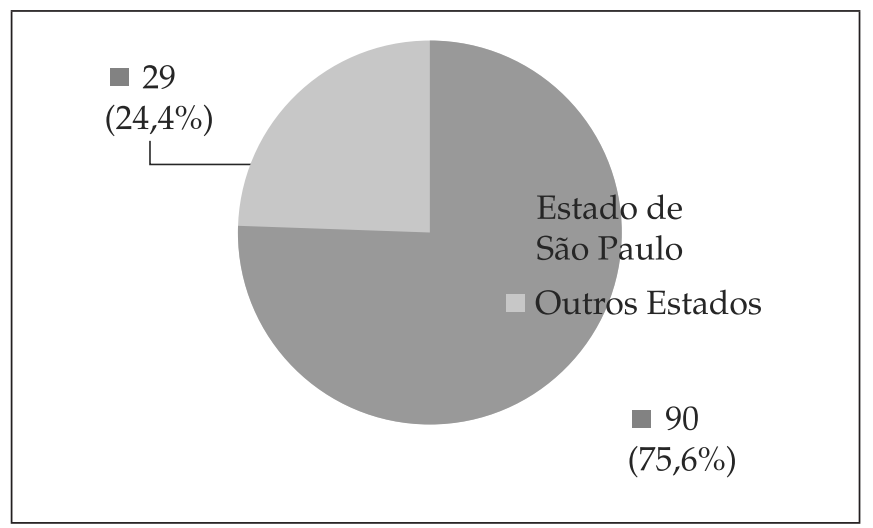

Dos 90 egressos nascidos no Estado de São Paulo, cerca de três quartos residem atualmente na cidade de São Paulo. Do restante, 19 pessoas moram em outros municípios deste Estado e 3 no exterior. Dentre os ex-residentes oriundos de outros Estados, 28/29 (96,5\%) permanecem morando e trabalhando no município de São Paulo.

Dos 97 egressos que responderam à pergunta sobre a motivação para a escolha da Pediatria como especialidade, as respostas mais citadas foram: amor ao trabalho com a criança e cuidado integral ao paciente (Tabela 1).

O programa de residência que experimentaram foi considerado adequado por $90 / 118(76,2 \%)$ respondentes, sendo que $28 / 118(23,7 \%)$ julgaram que ele poderia ser melhorado. Vinte e duas pessoas comentaram que a residência de Pediatria prepara o profissional preferencialmente para o atendimento de emergências e referiram que o preparo do futuro pediatra para o exercício profissional na clínica privada ficou aquém do esperado, principalmente no âmbito do atendimento de especialidades pediátricas. A carga de trabalho da residência de Pediatria foi considerada pesada, e o tempo livre para estudo e/ou pesquisa foi considerado escasso. Assim, obtivemos sugestões no sentido de dar mais ênfase ao treinamento para atendimento em clínica privada (10), aumentar o tempo livre para estudo e pesquisa (6) e oferecer maior exposição às especialidades pediátricas.
TABELA 1

Respostas relativas à motivação para a escolha da carreira profissional de pediatra

\begin{tabular}{ll}
\hline Motivação Referida & No (\%) \\
\hline Amor ao trabalho com crianças / Pediatria & $41(42,2)$ \\
Visão global do paciente & $25(25,7)$ \\
Especialidade generalista & $22(22,6)$ \\
Amor/empatia pela criança & $17(17,5)$ \\
Lidar com promoção e prevenção de saúde/crescimento & \\
e desenvolvimento/vida & $11(11,3)$ \\
Boa resposta da criança ao tratamento em relação ao & $10^{*}(10,3)$ \\
adulto & $8(8,2)$ \\
Interesse despertado durante a graduação & $6(6,2)$ \\
Aptidão pessoal & $3(3,1)$ \\
Lidar com a criança e sua família & $3(3,1)$ \\
Papel social do pediatra & $2(2,1)$ \\
Qualidade de vida & $2(2,1)$ \\
Atividade ambulatorial & $2(2,1)$ \\
Bom campo de trabalho &
\end{tabular}

Nota: *Destes, sete foram durante o Internato.

Os estágios cujas contribuições foram consideradas fundamentais à formação profissional se concentraram nas áreas de urgência/emergência, neonatologia e enfermarias de Pediatria geral e de especialidades pediátricas (aprovação superior a $90 \%$ ).

A grande maioria dos respondentes (99/118) acredita que o programa existente os preparou para enfrentar as ocorrências profissionais do cotidiano e também realizou algum curso com duração superior a três meses para treinamento e capacitação após o término da residência.

Quando perguntados sobre uma possível expansão do programa de residência de Pediatria para três anos, cerca de $72 \%(86 / 118)$ dos respondentes a consideraram uma alteração útil à melhoria da formação profissional. Sobre os estágios que julgavam os mais indicados para compor o currículo do terceiro ano de residência, responderam o seguinte: 37/56 (66\%) elegeram o ambulatório de especialidades pediátricas e práticas nas áreas de dermatologia, oftalmologia, otorrinolaringologia e saúde mental.

A distribuição dos respondentes segundo a fonte de renda atual é apresentada no Gráfico 2.

Quanto ao início da pós-graduação senso estrito, 50/111 já a iniciaram ou completaram (45 para mestrado e 5 para doutorado). O exercício de função docente faz parte da rotina de 54/118 (45,7\%) dos respondentes, em instituições públicas (70\%), privadas (11\%) ou em ambas (19\%). 


\section{GRÁFICO 2}

Distribuição dos respondentes em relação à principal fonte de renda referida

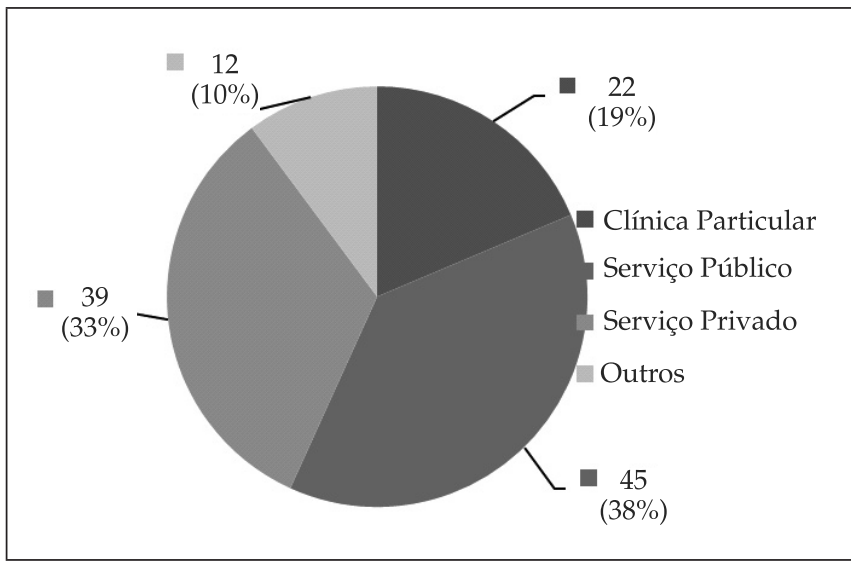

Em relação à expectativa profissional no momento, 75 egressos manifestaram vontade de iniciar ou continuar o curso de pós-graduação; 25 (33\%) buscar aprimoramento profissional $11(14,6 \%)$, aumentar o atendimento de consultório $10(13,3 \%)$, buscar maior reconhecimento profissional $6(8 \%)$, continuar e progredir nas atividades já iniciadas $3(4 \%)$, mudar para o interior ou mudar de Estado $2(2,6 \%)$, reduzir o número de plantões $2(2,6 \%)$ e dedicar mais a vida pessoal 1 $(1,3 \%)$. Dentre 100 respondentes, 78 se consideram profissionalmente realizados, e outros 22 não.

A maioria dos egressos refere que sua atuação profissional atual está concordante com o que havia planejado ao final da residência $(91 / 118)$. Para os que ainda se dizem insatisfeitos, os principais motivos são: o não envolvimento em projetos de pesquisa/programas de pós-graduação/vida acadêmica (10/27 - 37\%); não terem iniciado ainda clínica privada (3/27 - 10\%); apresentarem renda inferior ao esperado, necessitarem de muitos plantões para compor a renda mensal (2/27 - 7\%); e terem pouco tempo para dedicar à área de atuação escolhida $(1 / 27$ - 3\%). Somente um egresso abandonou a profissão, trocando-a pela indústria.

\section{DISCUSSÃO}

A Pediatria constitui uma especialidade clínica desde os anos 1900, tendo como missão a provisão de cuidado exemplar à saúde do recém-nascido, lactente, criança, adolescente e adulto jovem ${ }^{1}$.

Em 1978, foi criada a força-tarefa sobre o futuro da educação pediátrica (Fope I), sob os auspícios da Academia Americana de Pediatria. O primeiro relatório deste projeto apontou a necessidade de aumentar o tempo exercido pelo residente de Pediatria em nível ambulatorial, no sentido de aprimorar habilidades de treinamento nas questões de desenvolvimento, comportamento e do adolescente, no âmbito de uma equipe multidisciplinar de profissionais da saúde.

Desde então, a evolução do conhecimento médico e a necessidade eventual de redefinir o papel do pediatra no cuidado à criança e ao adolescente motivaram a revisão do documento inicial no ano de $1996^{2}$. Essa revisão destacou que as mudanças nos padrões de morbi-mortalidade infantil, a transformação sociodemográfica da população e o avanço tecnológico, principalmente em Biologia Molecular, Genética e Tecnologia da Informação, deveriam se acompanhar de uma atualização no perfil de formação do pediatra, para contemplar novas atribuições, competências e habilidades inerentes à atuação profissional no século XXI. Estas mudanças deveriam direcionar o pediatra geral para um maior envolvimento no cuidado de casos mais complexos e para a colaboração com o pediatra especialista no cuidado ao doente crônico. O pediatra especializado, além de prestar cuidado direto ao paciente e participar de atividades de ensino e pesquisa, continuaria a atuar como consultor do pediatra generalista, do especialista não pediatra, e adquiriria outras obrigações, como liderança no estabelecimento de protocolos clínicos de diagnóstico e seguimento, responsabilidade por orientações sobre políticas públicas de saúde aos órgãos públicos, agências internacionais e fundações. O avanço tecnológico das comunicações favoreceria o intercâmbio constante entre pediatras generalistas e especialistas tanto para a cobertura das necessidades rotineiras, como para o provimento de educação médica continuada.

A análise das manifestações de nossos egressos pode ser interpretada como um eco às conclusões deste relatório. Embora $76 \%$ dos respondentes considerem adequado o programa atual de residência médica, $72 \%$ pontuam que seria interessante aumentá-lo para três anos, justamente para aprofundar o treinamento nas especialidades pediátricas. $\mathrm{O}$ reconhecimento de que o programa de Residência Médica em Pediatria precisa ser expandido para três anos é pauta da Sociedade Brasileira de Pediatria desde 2007, uma vez que nosso país é o único da América do Sul em que ainda se formam pediatras em apenas dois anos de residência médica. Em todos os demais, o treinamento dura três anos.

Outro aspecto interessante demonstrado pelos respondentes é a tendência que o programa de residência médica tem de fixar o residente: nenhum dos respondentes originários das regiões Norte, Nordeste e Centro-Oeste retornou ao Estado natal. Este aspecto da residência médica foi reconhecido em nosso país e fundamentou a necessidade de implementar o Programa Nacional de Apoio à Formação de Médicos Espe- 
cialistas em Áreas Estratégicas (Pró-Residência), projeto dos ministérios da Saúde e Educação, com o apoio dos Conselhos dos Secretários Estaduais e Municipais de Saúde (Conass e Conasems), que oferece bolsas de residência médica em áreas definidas como prioritárias para o Sistema Único de Saúde (SUS) e carentes de determinados especialistas. O objetivo do programa é favorecer a formação de especialistas na modalidade de residência médica em regiões e especialidades prioritárias, definidas em comum acordo com os gestores do SUS. Podem enviar projetos os hospitais universitários federais e de ensino e secretarias de saúde, preferencialmente das regiões Norte, Nordeste e Centro-Oeste 9 .

Por outro lado, este estudo apresenta problemas característicos de estudos baseados em questionários, relacionados principalmente à baixa adesão à resposta, sendo que boa parte dos respondentes se constituiu de médicos atualmente ligados à nossa instituição por vínculo de trabalho ou de pós-graduação. Sabemos que as sugestões dos respondentes têm validade interna, pois são consonantes com o conteúdo dos fóruns que realizamos semestralmente com nossos residentes de primeiro e segundo ano. Nestes fóruns, os residentes apresentam suas opiniões sobre os estágios que executaram tanto com base em questionário estruturado - que aborda questões como conteúdo programático, excelência e compatibilidade na abordagem dos objetivos cognitivos, habilidades e atitudes, relacionamento com os preceptores e supervisores -, como de maneira aberta, em sessão plenária conjunta com os supervisores de cada estágio do programa. Fica, no entanto, a pergunta sobre a validade externa de nossos achados. Seria necessário aplicar este instrumento em outros serviços, idealmente em nível nacional, para que, a partir de resultados mais abrangentes, pudéssemos chegar a um consenso sobre o conteúdo programático necessário à formação do pediatra brasileiro.

Concomitantemente ao processo de redefinição dos objetivos da residência médica em Pediatria, a força-tarefa sobre o futuro da educação pediátrica está desenvolvendo um processo conjunto com o AGCME (Accreditation Council for Graduate Medical Education), órgão de acreditação dos serviços de residência médica nos Estados Unidos, no sentido de integrar a aquisição e a avaliação das competências básicas da residência médica. O programa tem como foco utilizar dados do desempenho evolutivo dos residentes dos programas de residência médica como base para a acreditação do programa formador. Parte deste banco de dados de desempenho incluiria indicadores de qualidade do serviço, questionários com pacientes, funcionários e alunos da graduação, além das notas dos residentes nos exames nacionais para obtenção de licença profissional.
Em conclusão, questionários eletrônicos aplicados pela internet constituem ferramenta prática e atual para obter informações dos egressos. A monitoração periódica de egressos de programa de residência é um instrumento muito útil para avaliação de programa e monitoramento das intervenções implementadas, pois certamente viabiliza a obtenção de informações atualizadas que ajudam no planejamento de novos cursos. As atividades de ensino nas especialidades pediátricas necessitam de revisão em seu conteúdo e forma. Para tanto, o aumento da duração da residência médica em Pediatria para três anos parece inevitável.

\section{AGRADECIMENTOS}

Este trabalho reflete o empenho do Conselho de Departamento de pediatria FMUSP e da Comissão de Residência Médica da Pediatria FMUSP para o aprimoramento contínuo da RM no Departamento de Pediatria da FMUSP. Importantes contribuições à ideia original deste trabalho foram introduzidas pelos preceptores do Instituto Regional de Educação Médica Faimer Brasil. Há que ser mencionado o esforço dos egressos respondentes para alavancar a participação de seus pares no questionário, principalmente por meio das redes sociais, sem o qual não teríamos conseguido $50 \%$ de adesão.

\section{REFERÊNCIAS}

1. Cone TE. History of American Pediatrics. Boston, MA: Little, Brown and Company; 1979.

2. Gruskin A, Williams RG, McCabe ERB, Stein F, Strickler J, Chesney RW, Mulvey HJ, Simon JL, Alden ER. FOPE II Final Report of the FOPE II Pediatric Subspecialists of the Future Workgroup Pediatrics. 2000;106(Suppl):1224-44.

3. Sampaio, SAP. "A Implantação da residência médica no Hospital das Clínicas: 40 anos de história" Residência Médica. São Paulo: FUNDAP; 1994.

4. Leone CR, Jacob CMA. Residência Médica no Departamento de Pediatria da FMUSP Sistema de avaliação. Pediatria (São Paulo).1997;19:164-9.

5. Freed GL, Dunham KM, Switalski KE. Douglas Jones M, Jr, McGuinness GA. Recently Trained General Pediatricians: Perspectives on Residency Training and scope of practice Pediatrics. 2009;123:S38-S43.

6. Focaccia R, Elias PM, Amato Neto V. Residência médica em doenças infecciosas e parasitárias no Hospital das Clínicas da Faculdade de Medicina da Universidade de Säo Paulo Rev Hosp Clin Fac Med Univ. 1988;43:171-5.

7. Maniglia, JV. Perfil do egresso da residência em otorrinolaringologia e cirurgia de cabeça e pescoço da Santa Casa de Franca, da Faculdade de Medicina de São José do Rio Preto e da Clínica Maniglia. Arq Ciênc Saúde. 2004;11:29-36 
8. Estado financia Residência, mas regula pouco a oferta de profissionais http://www.cremesp.org.br/library/modulos/centro_de_dados/arquivos/residencia_medica.pdf)

9. Brasil. Ministério da Educação. Residências em Saúde. Residência Médica. [online]. [acesso em 7 out. 2010]. Disponível em: http: / / portal.mec.gov.br/index.php?option=com_ content\&view $=$ article \&id $=12263 \&$ Itemid $=506$

\section{CONTRIBUIÇÃO DOS AUTORES}

Vera Hermina K Koch participou no planejamento, execução, análise e interpretação dos dados do estudo. Ulysses Doria Filho participou na concepção e desenho deste estudo, realizando a montagem e execução do projeto e interpretação dos dados assim como da redação do texto. Valdes Roberto Bollela participou na concepção e desenho do estudo, realizando a análise e interpretação dos dados assim como da redação do texto.

\section{CONFLITO DE INTERESSES}

Declarou não haver.

\section{ENDEREÇO PARA CORRESPONDÊNCIA}

Vera Hermina K Koch

Rua das Mangabeiras, 91 - apto 81

Santa Cecília - São Paulo

CEP 01233-010 SP

E-mail: vkoch@terra.com.br 